E3S Web of Conferences 5, 01002 (2015)

DOI: $10.1051 /$ e 3 sconf/ 20150501002

(C) Owned by the authors, published by EDP Sciences, 2015

\title{
Towards Identifying Contribution of Wake Turbulence to Inflow Noise from Wind Turbines
}

\author{
Bharat Raj Agrawal ${ }^{1, a}$, Aaron Rosenberg ${ }^{1}$, and Anupam Sharma ${ }^{1}$ \\ ${ }^{1} 2271$ Howe Hall, lowa State University, Ames, IA, 50011, USA
}

\begin{abstract}
Downstream turbines in a wind farm often operate under the influence of wakes from upstream turbines. Aerodynamic losses and aeromechanical issues (stochastic loads) associated with such wake-turbine interactions have been investigated before. However, the role such interactions play in the generation of aerodynamic noise has not been evaluated. This paper presents a two-step approach for predicting noise due to wake-turbine interaction. The first step involves an aerodynamic analysis of a wind farm using large eddy simulations. Time accurate data and turbulence statistics in the turbine wakes are obtained from this simulation just ahead of the downstream wind turbines. The second step uses the turbulence information with aeroacoustic models to predict radiated noise in the far field. Simulation results of two simplified model problems corresponding to these two steps are presented in this paper.
\end{abstract}

\section{Introduction}

Aerodynamic noise from wind turbines is generated at the rotor blades and is broadband in nature [1]. The turbulence in the incoming flow and the "self-generated" turbulence in the blade boundary layers are responsible for noise generation. These are referred to as "inflow turbulence" noise and "self" noise sources respectively. Recent research has focused predominantly on "self" noise from wind turbines, especially trailing edge noise. Relatively little work has been performed on inflow turbulence noise perhaps because of the community resigning to the belief that nothing can be done about it. An even less explored phenomenon is the acoustic impact of wake-turbine interaction and its contribution towards overall noise from wind turbines operating in a wind farm. This paper aims at assessing and quantifying the role of wake-turbulence in aerodynamic noise generation from wind turbines operating in a wind farm.

Aerodynamic interaction between turbines in wind farms is now relatively, though arguably, well understood. It is known that wakes from upstream turbines can significantly induce aerodynamic losses [2] and turbulent loads [3] on downstream turbines. These 'wake turbulence' induced aerodynamic losses and loads are prominent when a "stable" atmospheric condition is established over the wind site. High wind shear and low atmospheric turbulence are characteristic of stable atmosphere [4]. In these conditions, turbine wakes persist for long distances and hence wake-turbine interactions become more prominent. Low free-stream turbulence and increased wake-turbine interactions

ae-mail: bharatr@iastate.edu

This is an Open Access article distributed under the terms of the Creative Commons Attribution License 4.0, which permits unrestricted use, distribution, and reproduction in any medium, provided the original work is properly cited. 
associated with stable atmospheric conditions enhance the role of wake turbulence in noise generation. Furthermore, high velocity shear is conducive to the generation of other amplitude modulation (OAM), which has recently gathered tremendous attention $[5,6]$. Stable conditions also promote "channeling" of noise propagating in the downstream direction and hence cause more annoyance to wind farm neighbors.

\section{Numerical Predictions}

\subsection{Approach}

A schematic of the numerical noise prediction approach is shown in Fig. 1. It consists of two steps. In the first step, aerodynamics of a wind farm is simulated using a high-fidelity large eddy simulation (LES) methodology. The Simulator fOr WindFarm Applications (SOWFA) software is selected for this purpose. SOWFA can simulate aerodynamics of single or multiple turbines (windfarms) operating in uniform or atmospheric boundary layer flow. SOWFA uses the actuator line model [7] to parameterize the turbine rotors, which are represented by body force (sources) terms in the momentum equations. Time-accurate flow information and turbulence statistics are sampled from the LES simulations just ahead of the downstream turbines. The sampled data represents the flow that is ingested by the turbine.

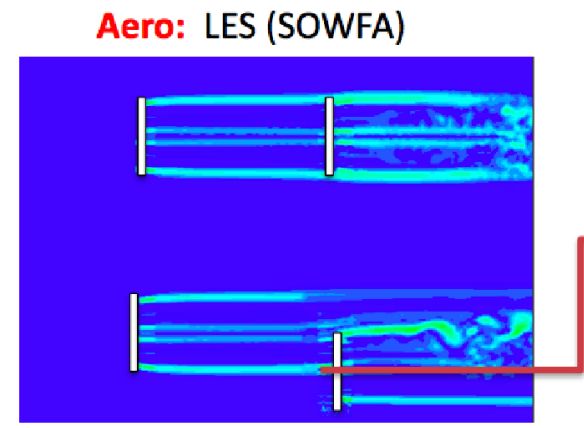

(a)



(b)

Figure 1: Envisioned approach for noise prediction: (a) Step 1, (b) Step 2.

The second step of the prediction approach can be performed using one of two methods. The first method is semi analytical. It uses the inflow turbulence intensity and the integral length scale, computed in the first step, with Lowson's formulation [8] to estimate the radiated noise. The second method involves another LES simulation where the interaction of the wake turbulence with the rotor blade (part span) is numerically solved to compute the noise. The inflow boundary condition for such LES simulations is set using the turbulence information extracted from the wind farm aerodynamics simulations performed in the first step.

Since the aerodynamic noise source in wind turbines is known to scale with flow velocity to the power $n$, where $n$ is between 5-6, only the outboard region of the rotor is important from the noise perspective. Therefore, a part-span simulation is performed with periodic boundary conditions in the spanwise direction. The inflow turbulence for this part-span simulation has to be acquired in a frame rotating with the turbine blades in the preceding (Step 1) aerodynamics computation. This turbulence 
is then specified over the mean blade-relative flow speed in this part-span LES calculation. The span length is chosen carefully to balance between computational power requirements and ensuring that the span length is larger than the coherence length. These part-span, blade-resolved LES provide unsteady pressure on the blade and other near field data which is combined with two different acoustic solvers to compute far-field noise.

The following sections describe results of two model problems corresponding to the two steps of the process.

\subsection{Wind Farm Aerodynamics}

SOWFA [3, 9] software is used to carry out wind farm aerodynamics simulations. In this LES model, spatially filtered, incompressible forms of continuity and Navier-Stokes equations are solved using spatial and temporal discretization. Spatial filtering introduces unresolved, sub-filter scale (also called sub-grid scale or SGS) stresses, which have to be modeled. The width of the spatial filter is taken to be the grid-filter width given by $\Delta=\left(\Delta_{x} \Delta_{y} \Delta_{z}\right)^{1 / 3}$. The actuator line model (ALM) is used for rotor parameterization so that the rotor blade geometry is not represented in the simulations. Denoting spatially-filtered quantities by ( ), the governing fluid flow equations are

$$
\begin{aligned}
\frac{\partial \widetilde{u_{i}}}{\partial x_{i}} & =0 \\
\frac{\partial \widetilde{u_{i}}}{\partial t}+\widetilde{u_{j}}\left(\frac{\partial \widetilde{u_{i}}}{\partial x_{j}}-\frac{\partial \widetilde{u_{j}}}{\partial x_{i}}\right) & =-\frac{\partial \widetilde{p^{*}}}{\partial x_{i}}-\frac{\partial \tau_{i j}}{\partial x_{j}}+v \frac{\partial^{2} \widetilde{u_{i}}}{\partial x_{j}^{2}} \\
& -\underbrace{f_{i} / \rho_{0}}_{\text {turbine force }}+\underbrace{\delta_{i 1} F_{P}}_{\text {driving pressure }}+\underbrace{\left.\delta_{i 3} g_{0} \widetilde{\theta}-\langle\widetilde{\theta}\rangle\right) / \theta_{0}}_{\text {buoyancy force }}+\underbrace{F_{c} \epsilon_{i j 3} \widetilde{u_{j}}}_{\text {coriolis force }}, \\
\frac{\partial \widetilde{\theta}}{\partial t}+\widetilde{u_{j}} \frac{\partial \widetilde{\theta}}{\partial x_{j}} & =-\frac{\partial q_{j}}{\partial x_{j}}+\alpha \frac{\partial^{2} \widetilde{\theta}}{\partial x_{j}^{2}},
\end{aligned}
$$

where, $\widetilde{p^{*}}=\widetilde{p} / \rho_{0}+\widetilde{u_{i}} \widetilde{u_{j}} / 2$ is the modified kinematic pressure, $\tau_{i j}=\widetilde{u_{i} u_{j}}-\widetilde{u_{i}} \widetilde{u_{j}}$, is sub-grid scale (SGS) stress tensor, and $q_{j}=\widetilde{u_{j} \theta}-\widetilde{u_{j}} \widetilde{\theta}$ is SGS heat flux. $f_{i}$ is momentum source to model forces exerted by turbine blades, $\delta_{i 1} F_{P}$ is pressure gradient to drive flow, $\theta$ is potential temperature and $\alpha$ is thermal diffusivity of the fluid. The deviatoric part of the SGS stress tensor, $\tau_{i j}$ is typically modeled using an eddy-viscosity model, $\tau_{i j}-1 / 3 \delta_{i j} \tau_{k k}=-2 v_{s g s} \widetilde{S_{i j}}$ and the SGS heat flux with an eddydiffusivity model $q_{j}=\widetilde{u_{j} \theta}-\widetilde{u_{j}} \widetilde{\theta}=-\left(v_{s g s} / P r_{s g s}\right) \partial \widetilde{\theta} / \partial x_{j}$, where, $\widetilde{S_{i j}}=1 / 2\left(\partial \widetilde{u_{i}} / \partial x_{j}+\partial \widetilde{u_{j}} / \partial x_{i}\right)$ is the resolved strain-rate tensor and $P r_{s g s}$ is the SGS Prandtl number. The mixing-length model by Smagorinsky [10], $v_{s g s}=\left(C_{S} \Delta\right)^{2}|\widetilde{S}|$ is used to model $v_{s g s}$. In the original model, $C_{S}$ was assumed to be a constant, but dynamic calculation of this coefficient has been used in recent years. Improved, tuning-free, scale-dependent SGS models have also been developed (see e.g., Ref. [2]) and used for atmospheric flow and wind plant simulations.

SOWFA uses a finite volume formulation and the discretization is second order accurate in space (central) and time (backward). A two-step solution procedure is used. In the first (precursor) step, the turbines are removed and turbulent flow in the domain is calculated. After the solution reaches a quasi-equilibrium state, time-accurate data is sampled at every time step on the "inlet" plane and stored. This data is specified as a boundary condition for the subsequent wind plant calculations. Other researchers $[11,12]$ have used periodic BCs with a buffer layer where the mean velocity is scaled to the desired vertical profile. 
Figure 2 (a) shows a schematic of the hypothetical wind farm for the first model problem. The wind farm has four wind turbines with two turbines (1 and 2) that experience freestream flow, and turbines 3 and 4 that operate in full and partial wakes of turbines 2 and 1 respectively. The simulation results shown here are for uniform inflow, instead of atmospheric boundary layer (ABL) flow. Therefore, turbines 1 and 2 have zero inflow turbulence. Figure 2 (b) shows iso-surfaces of vorticity magnitude. The iso-surfaces are drawn for vorticity values such that the helical tip vortex structure (trailing vorticity) in the turbine wake and the blade-bound vorticity are visible.

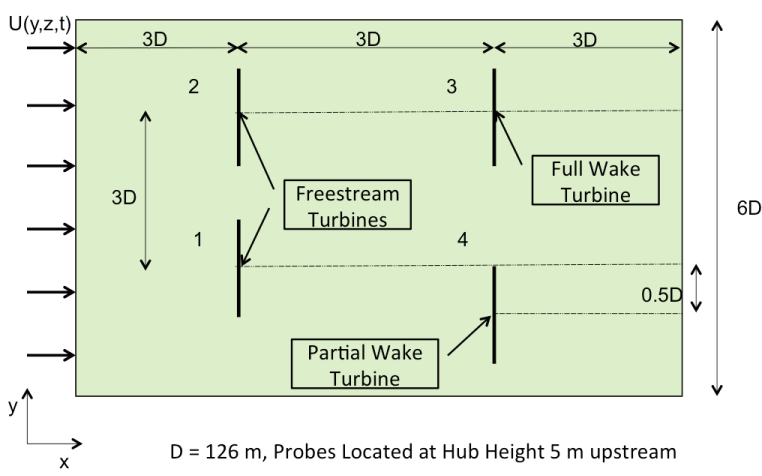

(a) Wind farm layout

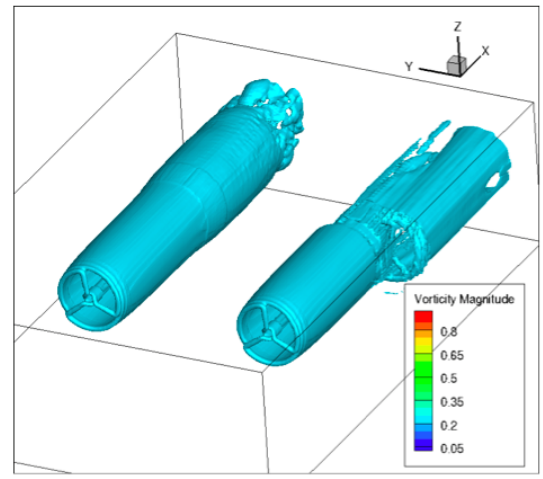

(b) Iso-surfaces of vorticity magnitude

Figure 2: Wind farm aerodynamics problem setup and results: (a) wind farm layout, and (b) isosurfaces of vorticity magnitude.



(a) Velocity contours

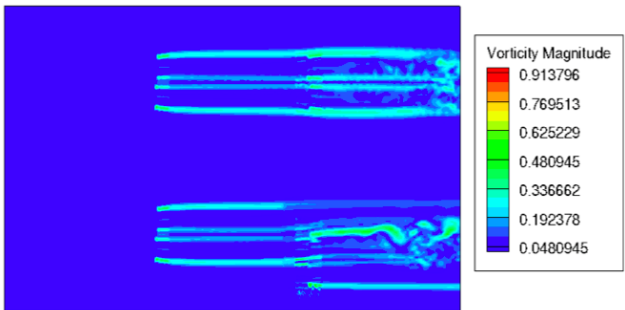

(b) Vorticity contours

Figure 3: Wind farm results showing (a) velocity magnitude and (b) vorticity in the horizontal plane at turbine hub height.

Flow turbulence data is extracted on a horizontal plane at the hub height. Figure 3 (a) and (b) show the contour plots for velocity magnitude and vorticity in this horizontal plane. It is seen that wake structure for turbines 3 and 4 differ significantly due to the difference in interactions with the upstream turbine wakes.

Complete time history of flow information at a point probe directly upstream of the hub of turbine 4 is also sampled. Figure 4 plots the streamwise velocity variation with time sampled at this point, and its autocorrelation function. The Autocorrelation function $\left(R_{u u}(\tau)\right)$ is used to compute the integral time scale $(T)$, which is subsequently used to compute the integral length scale $\left(l_{t}\right)$ using Taylor's 
frozen turbulence hypothesis.

$$
\begin{aligned}
R_{u u}(\tau) & =\frac{\langle u(t) u(t+\tau)\rangle}{\left\langle u^{2}(t)\right\rangle}, \\
T & =\int_{0}^{\infty} R_{u u}(\tau) d \tau, \\
l_{t} & =\langle U\rangle \times T
\end{aligned}
$$

where, $u=U-\langle U\rangle$ is the perturbation (mean-subtracted) stream-wise velocity.



(a) Temporal variation

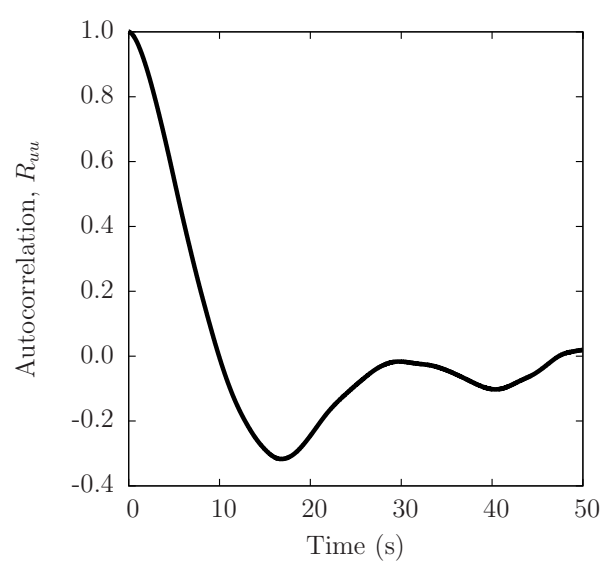

(b) Autocorrelation

Figure 4: Streamwise velocity component in the wake: (a) time variation, and (b) its autocorrelation.

\subsection{Noise Calculation: Semi-empirical approach}

Using the integral scales computed above, the far-field noise spectrum is computed using Eq. 2 due to Lowson [8]. Lowson's model (Eq. 2 is an extension of Amiet's theory [13] with a correction for low frequency noise estimation

$$
\begin{aligned}
\mathrm{SPL}_{1 / 3}^{H} & =10 \log _{10}\left[\left(\rho_{0} c_{0}\right)^{2} \frac{L}{2_{0}^{2}} l_{t} M^{3} I^{2} U_{0}^{2} \frac{K^{3}}{\left(1+K^{2}\right)^{-7 / 3}}\right]+58.4 \\
\mathrm{SPL}_{1 / 3}^{L} & =\mathrm{SPL}_{1 / 3}^{H}+10 \log _{10}(\underbrace{10 S^{2} M K^{2} /\left(1-M^{2}\right)}_{\text {low freq corr }})
\end{aligned}
$$

where, $I$ is turbulence intensity, $U_{0}$ is flow speed, $L$ is airfoil span, $K=\omega c /\left(2 U_{\text {rel }}\right)$ is wavenumber based on semi-chord $(c / 2)$ and $S^{2}$ is the compressible Sears function. Figure 5 shows the noise predictions for a few representative values of turbulent intensities and length scales. The spectra show little variation in noise in higher frequencies, however significant difference is observed in lowfrequency noise. 


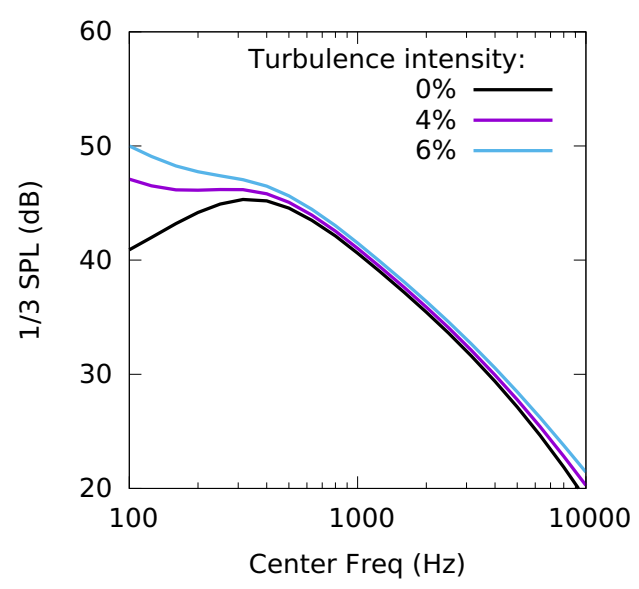

(a) Length scale, $\Lambda=7.5 \mathrm{~m}$

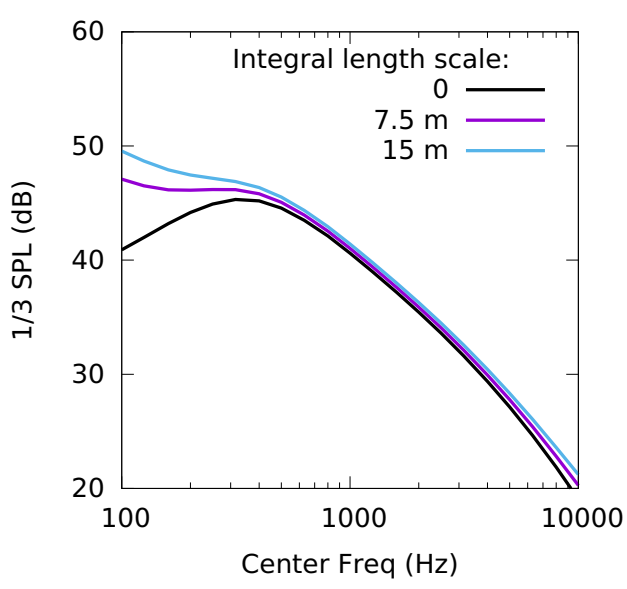

(b) Turbulence intensity, $\mathrm{TI}=4.0 \%$

Figure 5: Noise spectra for different (a) turbulent intensities and (b) integral length scales.

\subsection{Noise Calculation: LES approach}

A second, higher-fidelity method for noise prediction is sought that uses LES to model turbulenceairfoil interaction. As explained in Sec. 2.1, this would require using turbulence information from windfarm aerodynamics calculations and prescribing them as inflow boundary conditions for noise computation. This framework is not yet ready. We instead demonstrate the LES-based noise prediction methodology on a model problem where the turbulence is generate by another body. This model problem consists of a cylindrical rod placed upstream (in tandem) of an airfoil (NACA 0012). Wake from the rod impinges on the airfoil and produces unsteady lift that then radiates as noise.

\subsection{Rod-Airfoil Noise}

The problem setup and details of the simulation procedure for the rod-airfoil problem have been described elsewhere $[14,15]$ and hence the description is kept brief. Figure 6 (a) show a snapshot of the flowfield setup due to the rod-airfoil interaction. The rod wake comprises of quasi-periodic vortex shedding (peak Strouhal number, St $\sim 0.19$ ) and turbulence (due to vortex breakdown) which give tonal and broadband noise respectively. Two flow solvers are benchmarked against experiments: a compressible flow solver (Charles) by Cascade Technologies and an incompressible flow solver (PisoFOAM) from OpenFOAM. Flow Reynolds number based on the diameter of rod $\left(\operatorname{Re}_{\mathrm{d}}\right)$ is 48,000 and Mach number is 0.2. Figure 6 (b) show the schematic along with the positions of point probes A and $\mathrm{B}$ in the near field chosen for near-field spectral analysis.

Figures 7 and 8 compare the power spectral densities (PSDs) of velocity in the near field and sound pressure in the far field with experimental results [16]. Figure 7 compares the PSDs of streamwise velocity at the point probes $\mathrm{A}$ and $\mathrm{B}$ for both simulations. Figure 8 compares the far-field noise PSD at a point transverse of flow direction from the leading edge of the airfoil. Far-field noise PSD is computed from the solution of Charles simulation using the Ffowcs-Williams Hawkings acoustic analogy [17] and Amiet's theory [13]. Spectral prediction using both approaches match well with the 


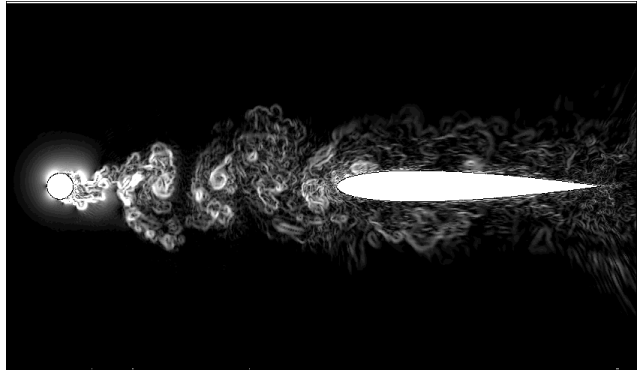

(a) Contours of $|\rho|^{1 / 4}$

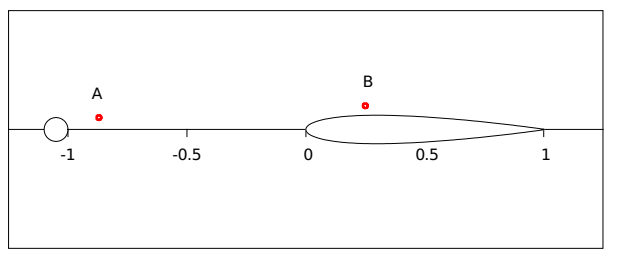

(b) $x / c=0.25$

Figure 6: Contours of $|\rho|^{1 / 4}$ in the flow field around rod-airfoil configuration and near-field probe locations.

experiments for peak (vortex shedding) frequency and amplitude. Agreement in the spectral decay at high frequencies is also acceptable.

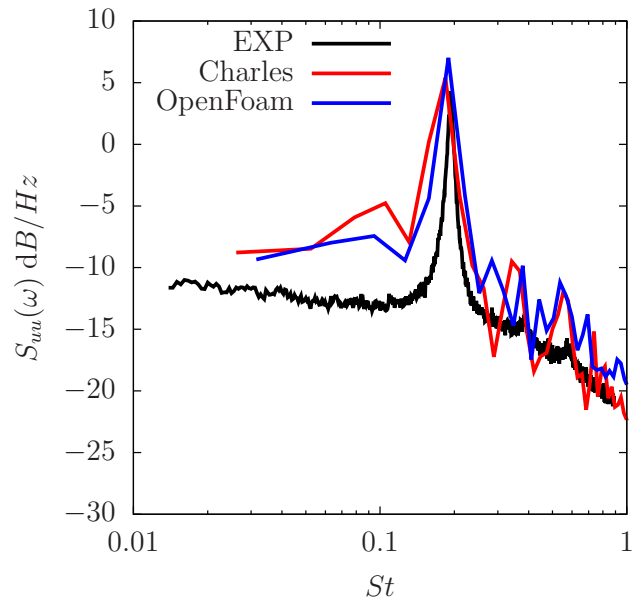

(a) $x / c=-0.87$

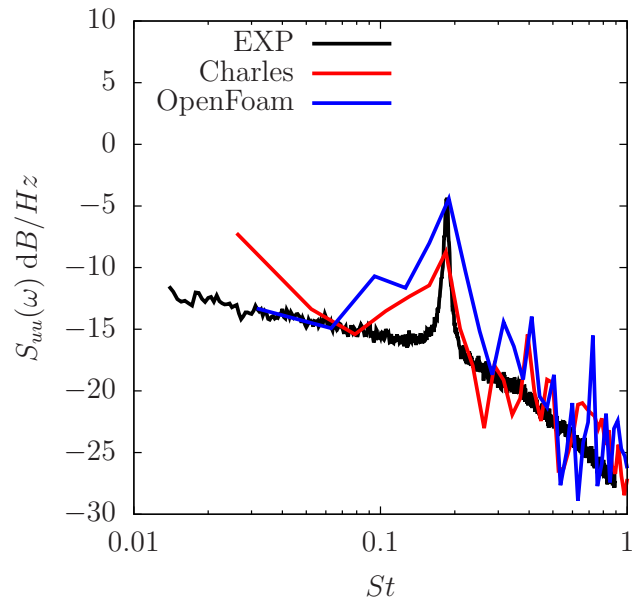

(b) $x / c=0.25$

Figure 7: Velocity power spectral density, $S_{u u}(\omega)$ at the two near-field probes indicated by $A$ and $B$ in Fig. 6 (b).

\section{Conclusion and Future Work}

An approach to assess the impact of turbine wake turbulence on wind turbine noise in wind farms is presented. Model problems are solved to assess the accuracy of the noise prediction methodology. Two LES solvers are benchmarked against experimental measurements for the rod-airfoil problem. The next set of simulations will involve more realistic effects such as atmospheric boundary layer (ABL) inflow for wind farm computations. Integrating the whole approach will create a framework 




(a) PSD

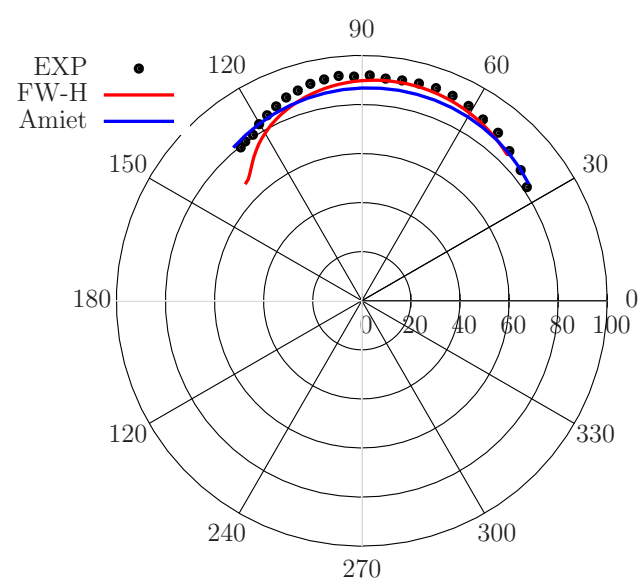

(b) Directivity

Figure 8: Far-field noise at a distance of 18.5 chords: (a) pressure spectral density (PSD) directly above the airfoil leading edge $\left(\theta=90^{\circ}\right)$ and (b) directivity. Predictions using the FW-H method [17] and Amiet's theory [13] are compared with measured data.

to study the effects such as relative importance of wake versus atmospheric (freestream) turbulence in producing inflow turbulence noise from wind turbines.

\section{Acknowledgment}

Funding for this research was provided by the General Electric Global Research Center and the Iowa Space Grants Consortium (Grant \#475-20-5). This work used the computational resources provided by the National Science Foundation Extreme Science and Engineering Discovery Environment (XSEDE) under Grant \#TG-CTS130004.

\section{References}

[1] S. Wagner, R. Bareiß, G. Guidati, Wind Turbine Noise (Springer-Verlag, 1996)

[2] F. Porté-Agel, Y. Wu, H. Lu, R.J. Conzemius, Journal of Wind Engineering and Industrial Aerodynamics 99 (2011)

[3] M. Churchfield, S. Lee, J. Michalakes, P. Moriarty, Journal of Turbulence 13 (2012)

[4] R.B. Stull, An Introduction to Boundary Layer Meteorology, Atmospheric and oceanographic sciences library (Kluwer Academic Publishers, 1988)

[5] D. Bowdler, Acoustic Bulletin of the Institute of Acoustics 33 (2008)

[6] S. Oerlemans, Wind Turbine Amplitude Modulation: Research to Improve Understanding as to its Cause $\mathcal{E}$ Effect, in RenewableUK report: Work Package A1 - An Explanation for Enhanced Amplitude Modulation of Wind Turbine Noise (2013)

[7] J.N. Sørensen, W.Z. Shen, Journal of Fluids Engineering 124, 393 (2002)

[8] M.V. Lowson, Assessment and Prediction of Wind Turbine Noise, in ETSU W/13/00284/REP (Flow Solutions Report 92/19, Briston, England, 1993) 
[9] M. Churchfield, S. Lee, P. Moriarty, A Large-Eddy Simulation of Wind-Plant Aerodynamics, in 50th AIAA Aerospace Sciences Meeting (Nashville, Tennessee, 2012)

[10] J. Smagorinsky, Monthly Weather Review 91 (1963)

[11] M. Calaf, C. Meneveau, J. Meyers, Physics of Fluids 22 (2010)

[12] Y. Wu, F. Porté-Agel, Boundary-Layer Meteorology 138 (2011)

[13] R.K. Amiet, Journal of Sound and Vibration 41 (1975)

[14] A. Sharma, Large Eddy Simulations for Predicting Aerodynamic Noise due to Rod Wake-Airfoil Interaction, in Conference on Wind Energy Science and Technology (Ankara, Turkey, 2013)

[15] B.R. Agrawal, A. Sharma, Aerodynamic Noise Prediction for a Rod-Airfoil Configuration using Large Eddy Simulations, in 20th AIAA/CEAS Aeroacoustics Conference (Atlanta, Georgia, USA, 2014)

[16] M.C. Jacob, J. Boudet, D. Casalino, M. Michard, Theoretical and Computational Fluid Dynamics 19, 171 (2005)

[17] J.F. Williams, D.L. Hawkings, 264, 321 (1969) 